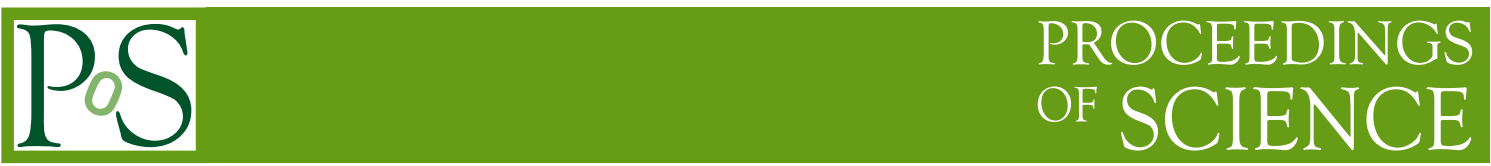

\title{
Density Imaging of Volcanoes with Atmospheric Muons using GRPCs
}

\author{
Cristina Cârloganu* \\ Clermont Université, Université Blaise Pascal, CNRS/IN2P3, Laboratoire de Physique \\ Corpusculaire, BP 10448, 63000 Clermont-Ferrand, France \\ E-mail: carloganein2p3.fr
}

\section{on behalf of the TOMUVOL Collaboration}

\begin{abstract}
Their capability to penetrate large depths of material renders high-energy atmospheric muons a unique probe for geophysical explorations. Provided the topography of the target is known, the measurement of the attenuation of the muon flux permits the cartography of matter density distributions revealing spatial and possibly also temporal variations in extended geological structures. A collaboration between volcanologists, astroparticle- and particle physicists, TOMUVOL, was formed in 2009 to study tomographic muon imaging of volcanoes with high-resolution tracking detectors. By exploiting Glass Resistive Plate Chambers (GRPCs) with a semidigital readout developped for ILC hadronic calorimetry within the CALICE collaboration, TOMUVOL aims to improve the understanding of volcanic processes and may finally contribute to reducing volcanic hazards. In 2011, TOMUVOL operated a muon telescope with three parallel detection planes at the flank of the Puy de Dôme, an inactive volcanic dome situated in the Massif Central (southcentral France). This contribution presents preparatory work towards muon tomography as well as flux measurements obtained after the first months of data taking.
\end{abstract}

The 2011 Europhysics Conference on High Energy Physics-HEP 2011,

July 21-27, 2011

Grenoble, Rhône-Alpes France

\footnotetext{
* Speaker.
} 


\section{Puy de Dôme as a reference site for volcano imaging with atmospheric muons}

Since high energy muons have a large penetrating power and a natural source exists for them, the atmospheric muons, they have been considered since a long time as a choice probe for large structures as the pyramids or for estimating the snow overburden on mountains [1]. Recent detector developments resulted in the availability of large area trackers with very fine segmentation, making possible to take one step further the muon imaging by applying it to km scale targets, as the volcanoes [2]. The basic idea behind muon imaging of volcanoes is that measuring the absorption of the muon flux as function of the direction at a fixed location permits mapping out the average column density in the volcano once the topography is known. By repeating the measurement from different locations, three-dimensional models of the matter density distribution can then be computed.

Precise deep volcano-imaging is one of main aims of the volcanologists currently, in their effort to better understand these complex objects, better quantify volcanic hazards, and make better risk predictions for crisis management in the 21 st century. The muon imaging, if successful to the required accuracy level (tens of metres of spatial resolution, few percent of density contrast) could locate and characterise in terms of size, depth and shape, magma reservoirs, volcanic conduits and hydrothermal systems, particularly when combined with other techniques used in volcanology, as seismic, electrical and electromagnetic tomography and satellite interferometry.

TOMUVOL is a common project of particle- and astroparticle physicists and volcanologists initiated in 2009 to provide the proof of principle for this new method by defining a reference site, the Puy de Dôme (alt. 1464 m a.s.l.), an extinct, 11000 years old [3] volcanic dome in the Massif Central (south-central France) [4]. The aimed for muon tomography will be compared and combined with gravimetric and electrical-resistivity measurements for realising a precise 3D density-map of the volcano. Obviously, this imposes also to define a robust, portable system for muon imaging of volcanoes from an existing particle physics tracker based on Glass Resistive Plate Chambers (GRPCs) [5], developed by the CALICE collaboration for the ILC hadronic calorimetry [6].

The Puy de Dôme was chosen because in addition to being conveniently close to the research laboratories involved in the project, it has a remarkable structure with two domes originating from two subsequent eruptions, which occurred within a short time interval. Its density structure is therefore expected to be complex and highly contrasted. The first results from an electrical resistivity measurement performed in May 2011 confirm these expectations.

In addition to the electrical resistivity measurement, the main goal of the TOMUVOL collaboration for 2011 is the realisation of a density radiography of Puy de Dôme. To this end, a muon telescope with three parallel detection planes was operated between January and July 2011 at the flank of the Puy de Dôme, muon flux measurements were performed and the data are currently being analysed. Since accurate topographical data are required to compute the density images, a LiDAR survey of the target and its surroundings was realised in March 2011. An overall precision of better than $10 \mathrm{~cm}$ on a $0.5 \mathrm{~m}$ grid was achieved for the digital elevation model.

We report here on the preparatory work and on the first measurements taken at the Puy de Dôme. Section 2 is dedicated to the detection site and to the experimental setup. The preliminary results obtained after these first 7 months of data taking are presented in Section 3. Based on them, the prospects of muon imaging are evaluated in the concluding section. 


\section{The muon telescope and the detection site}

The TOMUVOL muon telescope is made of three parallel planes of GRPCs, operated in avalanche mode, with the high voltage (typically $7 \mathrm{kV}$ ) being adapted to environmental pressure and temperature conditions. A single chamber consists of two parallel thin glass plates kept at a distance of about $1 \mathrm{~mm}$ using tiny ceramic balls as spacers so that gas (93\% forane, 5\% isobutane, $2 \%$ SF6, with a throughput of $\sim 1$ liter $/ \mathrm{h}$ ) can circulate between the plates. The outer sides of the glass plates are coated with a thin layer of highly resistive material on which high voltage is applied. A thin Mylar layer serves as insulation between the anode and a layer of copper cells of $1 \mathrm{~cm}^{2}$ size assembled on one face of Printed Circuit Boards (PCBs) of $50 \times 33.3 \mathrm{~cm}^{2}$, assembled in $1 \mathrm{~m}^{2}$ slabs. The chambers are embedded in steel cassettes and are vertically mounted onto a movable aluminum support framework.

The readout ASICs (HARDROC2 [7]) handle each $8 \times 8$ pads. In total 48 ASICs are connected on one $1 \mathrm{~m}^{2}$ PCB-slot, leading to a total of 9142 readout channels per $\mathrm{m}^{2}$. For each pad, two ASIC-implemented comparators provide amplitude information for the digitised data. In datataking mode, each ASIC buffers up to 512 successive signals above the low threshold. They are read out through a Field Programmable Gate Array (FPGA) upon receival of an external, periodical trigger signal. The FPGAs manage also the communication with a PC through an USB interface. The data acquisition and monitoring software is customized for the experiment based on the crossplatform, distributed framework XDAQ [8]. The whole setup is controlled remotely through a long-range WIFI network. This link is also used to stream the recorded data to a central server. A video camera surveys the installation at all hours.

For the first radiographic measurements, the muon detector has been installed $600 \mathrm{~m}$ below the summit at a distance of $2 \mathrm{~km}$. An underground site in a basement was chosen to reduce the impact of low-energy background. To protect the electronics against moisture, the detector was placed under a tent of polyethylene foil. Additionally, a dehumidifier ventilates dry air under the tent. Environmental data (i.e. temperature, pressure and humidity) are recorded every 15 min and are archived in a data base.

From January to April 2011 data have been taken continuously with two $1 \mathrm{~m}^{2}$ chambers supplemented by a third chamber of $1 / 6 \mathrm{~m}^{2}$ size. The distance between the first and the second chamber was $49 \mathrm{~cm}$, the one between the second and the third, smaller chamber, $9 \mathrm{~cm}$. For three more weeks, the small chamber was replaced with a $1 \mathrm{~m}^{2}$ chamber and the distance between the outer chambers increased to $1 \mathrm{~m}$. In a third data taking period, which lasted until July 2011, the detector reverted to the original configuration, with two large chambers and a small one. A $1 \mathrm{~m}$ distance between the two outer chambers was however maintained.

In a right coordinate system with the origin set by the detector and with the observation direction defined by the elevation $\alpha$ and an angle $\beta$ in the horizontal plane such that $\beta=0$ indicates the summit of Puy de Dôme, the integrated path length that the atmospheric muons would have to travel through the volcano in order to reach the detector is represented in Fig. 1, left. The geometrical acceptance of each setup was evaluated with a ray-tracing simulation which takes into account missing channels: over the small solid angle over which the target is observed, the geometrical acceptance of the detector is maximal. The first performed simulations indicate that the Puy de Dôme will be accessible for muon imaging with an $1 \mathrm{~m}^{2}$ large detector. 

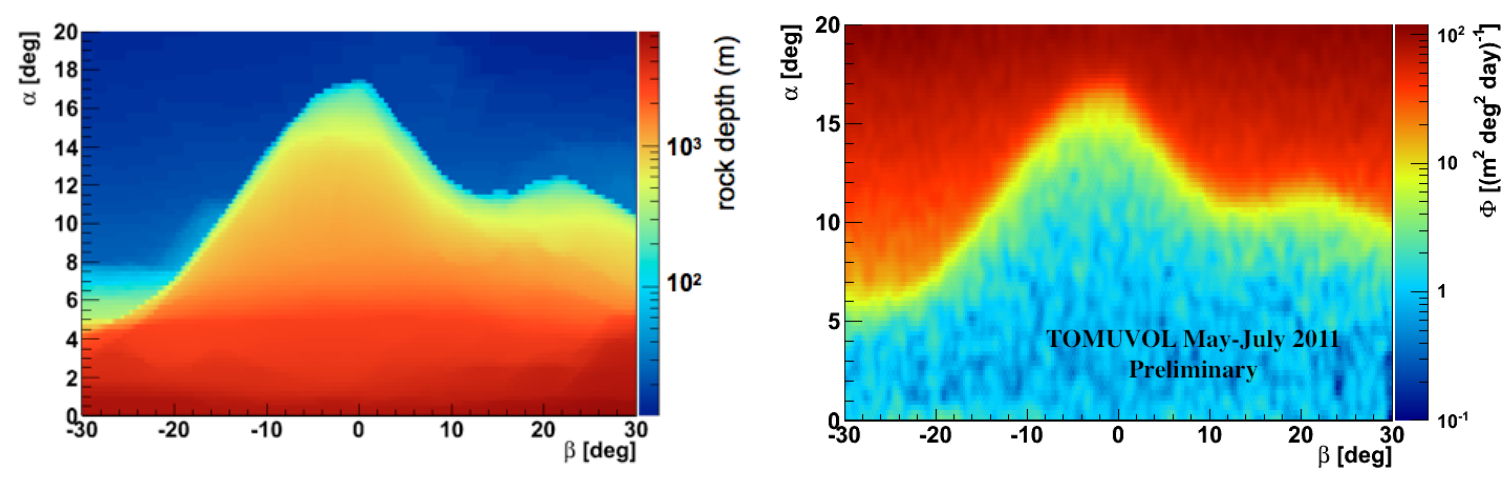

Figure 1: Left: Integrated path length that the atmospheric muons would have to travel through the volcano in order to reach the detector. Right: Shadow cast in the atmospheric muon flux by the Puy de Dôme, as measured with the second setup $\left(\mathscr{A}=1 \mathrm{~m}^{2}, 1 \mathrm{~m}\right.$ long).

During the data taking period, more than 15 million muon tracks from the entire sky have been recorded. The duty cycle reached almost 90 percent with only a few short interruptions for detector maintenance operations and systematic tests.

\section{Track reconstruction and first results}

The reconstruction algorithm preselects coincident hits in the three detection planes in a $0.4 \mu \mathrm{s}$ time window. The tracks are obtained analytically through a straight line minimisation procedure, which allows for track bundles. The overall $\chi^{2}$-distribution of the track residuals agrees well with the expectation. Overall, a position resolution of $0.4 \mathrm{~cm}$ and an angular resolution better than $0.5^{\circ}$ in both $\alpha$ and $\beta$ are achieved for the muon tracks during the first data taking period. The angular resolution is improved by about a factor 2 for the $1 \mathrm{~m}$-long setups.

To exploit this precision, the muon detector has been aligned with respect to the volcano with a $5 \mathrm{~mm}$ accuracy through GPS and tachymetric measurements. Additionally, the alignment of the detection planes has been checked with the tracks themselves by minimising the sum of the individual-track $\chi^{2} \mathrm{~s}$ against variations in the alignment parameters.

The shadow cast in the flux of atmospheric muons by the Puy de Dôme, as measured with the second setup $\left(\mathscr{A}=1 \mathrm{~m}^{2}, 1 \mathrm{~m}\right.$ long), is shown in Fig. 1, right. For this measurement, the event rates were corrected both for the detector acceptance and dead time. Note that a smoothing algorithm has been applied to the recorded map in order to reduce artificial bands (Moiré-patterns) typical of digital images. Due to the short exposure time of the data set, the significance of the flux through the volcano is statistically limited to regions close to the surface. The observed shape is in good agreement with the actual outline of the volcano. The full statistics accumulated until July 2011 is currently being analyzed in order to extract the first radiographic density distributions.

\section{Conclusion}

The TOMUvol collaboration explores tomographic imaging of volcanoes with atmospheric muons. For this purpose, a GRPC based prototype detector was installed at the flank of the Puy 
de Dôme in January 2011. Preparatory work for the computation of a tomographic density map, including a precise topographical LIDAR survey of the volcano, has been performed: the detector has been carefully positioned w.r.t. the volcano and its performance for in situ operation characterised. Algorithms for track reconstruction, detector alignment and image reconstruction have been developed and are now applied to first data.

The first seven months of in situ operation of the prototype detector showed that GRPCs are well suited for volcano imaging. Technical improvements to enhance the mobility of the detection setup were identified and allowed us to design a telescope that could be used also for active volcanoes under even more challenging environmental conditions. The analysis of the recorded data is underway and should provide soon a first radiographic density map of the Puy de Dôme, which will be compared and combined with images of the volcano's interior obtained through gravimetric and electrical tomographies.

The TOMUVOL collaboration acknowledges support from the University Blaise Pascal of Clermont-Ferrand, CNRS, Région Auvergne, Conseil Général du Puy-de-Dôme. We also thank Mr. Dreher, owner of the Grotte Taillerie du Puy-de-Dôme, who kindly hosted the Tomuvol detector during seven months.

\section{References}

[1] E.P. George, Commonwealth Engineer (July 1955), 455

L.W. Alvarez et al., Science 167, (1970), 832

[2] K. Nagamine et al., Proc. Jpn. Acad., Ser B 80 (2004), 179

[3] D. Miallier et al., C.R. Geoscience 342 (2010), 847-854

[4] P. Boivin et al., Volcanologie de la Chaîne des Puys, 5th ed. (2009)

[5] M. Bedjidian et al, JINST 6:P02001, 2011

[6] see https://twiki.cern.ch/twiki/bin/view/CALICE/WebHome

[7] N. Seguin-Moreau, Proc. of TWEPP Prague (2007) and IEEE Hawei (2007)

[8] see https://svnweb.cern.ch/trac/cmsos 